\title{
POSITIVE SEMIDEFINITE DIAGONAL MINUS TAIL FORMS ARE SUMS OF SQUARES
}

\author{
CARLA FIDALGO AND ALEXANDER KOVAČEC
}

\begin{abstract}
By a diagonal minus tail form (of even degree) we understand a real homogeneous polynomial $F\left(x_{1}, \ldots, x_{n}\right)=F(\underline{x})=D(\underline{x})-T(\underline{x})$, where the diagonal part $D(\underline{x})$ is a sum of terms of the form $b_{i} x_{i}^{2 d}$ with all $b_{i} \geq 0$ and the $\operatorname{tail} T(\underline{x})$ a sum of terms $a_{i_{1} i_{2} \ldots i_{n}} x_{1}^{i_{1}} \ldots x_{n}^{i_{n}}$ with $a_{i_{1} i_{2} \ldots i_{n}}>0$ and at least two $i_{\nu} \geq 1$. We show that an arbitrary change of the signs of the tail terms of a positive semidefinite diagonal minus tail form will result in a sum of squares of polynomials.
\end{abstract}

\section{Introduction}

The problem of the representability of positive semidefinite (psd) polynomials as sums of squares of polynomials (sos) has been treated by many authors. The first significant publication prompted by a question of Minkowski seems to be Hilbert's article [Hi] where it is shown that not all psd forms are sos. The first explicit examples for this phenomenon were given by Motzkin [Mo] in 1967 and shortly after by Robinson [Ro].

In the nineteen eighties and nineties this area was very much advanced by Reznick, partly in collaboration with Choi and Lam. Note that all psd polynomials are necessarily of even degree. One of Reznick's results [Re1] is that the form $F(\underline{a}, \underline{x})=a_{1} x_{1}^{2 d}+\ldots+a_{n} x_{n}^{2 d}-2 d x_{1}^{a_{1}} \ldots x_{n}^{a_{n}}$ can be written as a sum of at most $3 n-4$ squares of polynomials. With more squares allowed, $F(\underline{a}, \underline{x})$ can be written even as a sum of binomial squares (sobs), i.e. as sum of expressions of the form $\left(\alpha x_{1}^{i_{1}} x_{2}^{i_{2}} \cdots x_{n}^{i_{n}}-\beta x_{1}^{j_{1}} x_{2}^{j_{2}} \cdots x_{n}^{j_{n}}\right)^{2}$, see [Re2] (where always $\alpha=\beta$ ). If $\alpha$ or $\beta$ is 0 , these forms are squares of monomials.

Similar but much less economic representations than Reznick's had been found earlier by Hurwitz's [Hu]; see e.g. [BB, p8]. Reznick's paper [Re2] undertakes a profound investigation of forms obtained from the arithmetical geometrical inequality by substitution of its variables by monomial squares. He calls these forms agiforms.

\footnotetext{
Received December 31, 2008.
} 
We use one of his results on 'Hurwitz agiforms' to show that all psd diagonal minus tail (dmt) forms are sums of binomial and monomial squares. The psd dmt forms have the aspect $F\left(x_{1}, \ldots, x_{n}\right)=F(\underline{x})=D(\underline{x})-T(\underline{x})$, where the $d i$ agonal part $D(\underline{x})$, and tail $T(\underline{x})$ are defined via writing $a_{\underline{i}} \underline{\underline{i}}=a_{i_{1} \ldots i_{n}} x_{1}^{i_{1}} \ldots x_{n}^{i_{n}}$, by

$$
D(\underline{x})=\sum_{i=1}^{n} b_{i} x_{i}^{2 d}, \quad \text { and } \quad T(\underline{x})=\sum_{\underline{i} \in I} a_{\underline{i}} \underline{x}^{\underline{i}}, \quad d \in \mathbb{Z}_{\geq 1}, b_{i}, a_{\underline{i}} \geq 0,
$$

and $I \subseteq\left\{\underline{i}=\left(i_{1}, \ldots, i_{n}\right) \in \mathbb{Z}_{\geq 0}^{n}: 0 \leq i_{1}, \ldots, i_{n} \leq 2 d-1, i_{1}+\ldots+i_{n}=2 d\right\}$. Call a dmt-form elementary if its tail consists of at most one term. Such forms are generalizations of Reznick's agiforms on Hurwitz trellises in that the coefficients in the diagonal part are not necessarily the powers occurring in the tail term and its coefficient not necessarily equals the sum of the diagonal coefficients.

The main contribution is to adapt Reznick's idea to prove in theorem 2.3 that elementary psd dmt forms are sobs and theorem 2.7 saying that a psd dmt form $F$ can be written as a sum of elementary psd dmt forms. Consequently all psd dmt forms are sobs. As a byproduct we also obtain an easy proof that a psd dmt form restricted to the standard simplex has exactly one local minimum, see corollary 2.8. Other corollaries are corollary 2.9 saying that the sobs property persists even after changing the signs of the tail terms of a psd dmt form; and corollary 2.10 - a result due to Robinson - according to which adding $\beta\left(x_{1}^{2 d}+\ldots+x_{n}^{2 d}\right)$ to any form of degree $2 d$ will for large enough $\beta$ result in a sobs. We obtain this result in completely a different manner from Robinson's.

In section 3 the findings are put together in algorithm 3.1 for writing a psd dmt form as a sobs. We work an example for illustration. We also present examples due to Robinson, and Choi and Lam showing that in general it is false that a psd form even if having prominent diagonal part is sos.

For following the proofs of lemma 2.2 and theorem 2.3, and the algorithm and example in section 3, availability of [Re2] will be necessary; otherwise the essence of the paper can be understood without specialized knowledge. 


\section{Results}

If context avoids confusion, we write an n-tuple $\left(x_{1}, \ldots, x_{n}\right)$ often as $\underline{x}$. For parts of $\underline{x}$, like $\left(x_{1}, \ldots, x_{k}\right),\left(x_{k+1}, \ldots, x_{n}\right)$, we may write $\underline{x}_{1: k}, \underline{x}_{k+1: n}$, respectively. Special vectors we use are the standard vectors $\underline{e}_{i}=(0, \ldots, 0,1,0, \ldots, 0)$, with a single 1 at positions $i=1, \ldots, n$, and their sum $\underline{1}_{n}=(1,1, \ldots, 1) \in \mathbb{R}^{n}$. Consider now a polynomial $P(\underline{x})=\sum_{i \in I} c_{\underline{i}} \underline{x}^{\underline{i}} \in \mathbb{R}[\underline{x}]$. We say (quite naturally) that a term $c_{\underline{i}} \underline{x}^{\underline{i}}$ occurs in $P$ if $c_{\underline{i}} \neq 0$, a variable $x_{j}$ occurs in a such a term if $i_{j} \geq 1$, and the variable occurs in $P$ is there exists a $c_{\underline{i}} \neq 0$ such that $i_{j} \geq 1$. Denoting by $\operatorname{var}(P)$ the set of variables occurring in $P$, the dimension of $P$ is $\operatorname{dim}(P)=\# \operatorname{var}(P)$. A representation of a homogeneous polynomial (or form) of degree $\geq 1$ as a sum $P=P_{1}+\ldots+P_{k}$ with nonzero forms $P_{i}$, so that for all $1 \leq i<j \leq k, \operatorname{var}\left(P_{i}\right) \cap \operatorname{var}\left(P_{j}\right)=\emptyset$ will be called a decomposition of $P$. If such a representation forces $k=1, P$ is indecomposable. The decomposition is complete if the $P_{i}$ are indecomposable. A complete decomposition is easily seen to be unique up to the order of the summands $P_{i}$.

For example, $3 x_{1} x_{2}-x_{2} x_{3}+7 x_{4} x_{5}=P_{1}+P_{2}$ is a complete decomposition with indecomposable $P_{1}=3 x_{1} x_{2}-x_{2} x_{3}$ and $P_{2}=7 x_{4} x_{5}$. It is convenient to assign the zero polynomial the degree of the set of polynomials which we work with. This degree will be fixed and assumed positive throughout.

We begin with a simple lemma. It concerns variables and decomposability of forms, in particular psd forms and dmt forms. Its part b explains why much of the theory can be reduced to the investigation of indecomposable forms.

Lemma 2.1. a. If $F=D-T$ is psd dmt, then $\operatorname{var}(T) \subseteq \operatorname{var}(D)$.

$\mathrm{b}_{\mathrm{psd}} \& \mathrm{~b}_{\mathrm{dmt}}$. If $F=F_{1}+F_{2}+\ldots+F_{k}$ is a decomposition of a psd form (dmt form), then $F_{1}, F_{2}, \ldots, F_{k}$ are psd forms (dmt forms).

c. Assume $F=\sum_{i \in I} c_{\underline{i}} \underline{\underline{i}}$ is any form and $F=F_{1}+F_{2}+\ldots+F_{k}$ a decomposition. Let $\tilde{F}$ be obtained from $F$ by altering the signs of some of its terms: $\tilde{F}=\sum_{i \in I} \varepsilon_{\underline{i}} c_{\underline{i}} \underline{x}^{\underline{i}}$, with $\varepsilon_{\underline{i}} \in\{-1,+1\}$. Let $\tilde{F}_{i}$ be obtained from $F_{i}$ by giving each term in $F_{i}$ the sign it has in $\tilde{F}$. Then $\tilde{F}=\tilde{F}_{1}+\tilde{F}_{2}+\ldots+\tilde{F}_{k}$ is a decomposition of $\tilde{F}$.

Proof. a. Assume a variable, $x_{1}$ say, occurs in $\operatorname{var}(T)$ but not in $\operatorname{var}(D)$. Then $-T\left(x_{1}, 1, \ldots, 1\right) \rightarrow-\infty$ as $x_{1} \rightarrow \infty$, while $D\left(x_{1}, 1, \ldots, 1\right)=\sum_{i=2}^{n} b_{i}$ remains 
constant. Hence $F\left(x_{1}, 1, \ldots, 1\right)<0$ for large enough $x_{1}$, contradicting psdness of $F$. The other variables can be treated similarly.

$\mathrm{b}_{\text {psd }}$. Consider a decomposition of a psd form $F$ into two parts first: $F=$ $F_{1}+F_{2}$. Assume, say, $\operatorname{var}\left(F_{1}\right)=\left\{x_{1}, \ldots, x_{k}\right\}, \operatorname{var}\left(F_{2}\right)=\left\{x_{k+1}, \ldots, x_{n}\right\}$. Then $0 \leq F\left(\underline{0}_{1: k}, \underline{x}_{k+1: n}\right)=F_{1}(\underline{0})+F_{2}\left(\underline{x}_{k+1: n}\right)=F_{2}\left(\underline{x}_{k+1: n}\right)$. Hence $F_{2}$ is psd. Similarly $F_{1}$ is psd. The general case follows by induction. This result can also be seen as a special case of a result involving the Newton polytope of a psd polynomial: see e.g. theorem 3.6iv [Re2, p442].

$\mathrm{b}_{\mathrm{dmt}}$. Let $F=D-T$ be dmt and again consider a decomposition $F=F_{1}+F_{2}$ into two parts first. Since the family of nonzero terms in a decomposition $F_{1}+F_{2}$ of $F$ is precisely the disjoint union of the terms in $F_{1}$ with the terms in $F_{2}$, we can write $F_{1}=D_{1}-T_{1}$ and $F_{2}=D_{2}-T_{2}$, where $T=T_{1}+T_{2}$, $D=D_{1}+D_{2}$. It follows that $F_{1}, F_{2}$ are dmt forms. The general case follows by induction.

c. Is immediate.

For lemma 2.2, the reader will have to absorb especially the following pieces from Reznick's long paper [Re2]: framework and trellis [p433c7] (page 433, about 7 centimeters from the first textrow), $C(\mathcal{U}), E(\mathcal{U}), \bar{A}(\mathcal{B})$, definition of (simplicial) agiforms $f(\mathcal{U}, \underline{\lambda}, \underline{w})$ [p434c8], the Hurwitz trellis [p435c-5, $\mathrm{p} 436 \mathrm{c} 1], \mathcal{U}$-mediated sets [p438c3], the definition of $\mathcal{U}^{*}$, theorem 2.2 [p438], theorem 2.8 [p440], H-trellis [p439c2], and theorem 4.4 [p443c-5]. The following is proved but enunciated slightly weaker as corollary 4.10 in $[\operatorname{Re} 2$, p444].

Lemma 2.2. Every simplicial agiform $f(\mathcal{U}, \underline{\lambda}, \underline{w})$ on a H-trellis $\mathcal{U}$ is a sum of $\left|\mathcal{U}^{*} \backslash \mathcal{U}\right|$ binomial squares.

Proof. Let $f(\mathcal{U}, \underline{\lambda}, \underline{w})=\lambda_{1} \underline{x}^{\underline{\underline{u}}}+\ldots+\lambda_{m} \underline{x}^{\underline{u}_{m}}-x^{\underline{w}}$ be a simplicial agiform on a H-trellis. Then $\underline{w} \in C(\mathcal{U})=\mathcal{U}^{*}$. By Reznick's theorem 2.2, $\mathcal{U}^{*}$ is $\mathcal{U}$-mediated. Then his theorem 4.4 says that $f$ is a sum of $\left|\mathcal{U}^{*} \backslash \mathcal{U}\right|$ binomial squares.

In [Re1] it is shown that for the special case of the Hurwitz trellis $\mathcal{U}=$ $\mathcal{H}_{n, 2 d}=\left\{2 d \underline{e}_{i}: i=1, \ldots, n\right\}$ which is the one that interests us here most, one can obtain representations of less (but not necessarily binomial) squares. See our section 3 for some remarks on this. 
Note that the form $E(\underline{x})$ figuring in the next theorem is elementary dmt if and only if $\mu \geq 0$. Also note that dimension $n=1$ would not even allow us to speak of $a_{i}$ or $\mu$ since the form would collaps to a form $b_{i}^{\prime} x_{i}^{2 d}$.

Theorem 2.3. Let $n \geq 2, b_{1}, \ldots, b_{n} \in \mathbb{R}_{\geq 0}, d \in \mathbb{Z}_{\geq 1}$, and assume

$$
E(\underline{x})=b_{1} x_{1}^{2 d}+\ldots+b_{n} x_{n}^{2 d}-\mu x_{1}^{a_{1}} \cdots x_{n}^{a_{n}}
$$

to be an indecomposable form of dimension $n$. Then all $a_{i} \in \mathbb{Z}_{\geq 1}$. Hence

$$
\mu_{0}=2 d \prod_{i=1}^{n}\left(\frac{b_{i}}{a_{i}}\right)^{a_{i} / 2 d}
$$

is well defined and we have:

a. The following are equivalent:

i. $E$ is psd.

ii. $\mu \leq \mu_{0}$ and all $a_{i}$ are even or $|\mu| \leq \mu_{0}$ and some $a_{i}$ are odd.

iii. $E$ is sobs and hence sos.

b. If $\mu=\mu_{0}$, then $E \mid \mathbb{R}_{\geq 0}^{n}$ has (projectively counted) precisely one zero, namely at the point

$$
\left(\frac{a_{1}}{b_{1}}\right)^{1 / 2 d}:\left(\frac{a_{2}}{b_{2}}\right)^{1 / 2 d}: \ldots:\left(\frac{a_{n}}{b_{n}}\right)^{1 / 2 d} .
$$

Proof. Since $\operatorname{dim}(E)=n$, every $x_{i}, i=1, \ldots, n$, occurs in $E$. Also $n \geq 2$ implies $\mu \neq 0$, for otherwise the form would be decomposable. If $x_{1}$, say would not occur in $\mu \underline{x}^{\underline{a}}=\mu x_{1}^{a_{1}} \cdots x_{n}^{a_{n}}$, then $a_{1}=0$. So $b_{1} x_{1}^{2}+\left(\sum_{i=2}^{n} b_{i} x_{i}^{2}-\mu \underline{x}^{\underline{a}}\right)$ would be a decomposition of $E$, contradicting the hypothesis. Similarly for any other $x_{i}$. We conclude that all $a_{i} \in \mathbb{Z}_{\geq 1}$. Since all $b_{i} \geq 0$, the expression for $\mu_{0}$ is well defined.

$\mathrm{i} \Rightarrow$ ii. Let $E$ be psd. Assume first that some $b_{i}$ are 0, w.l.o.g. $b_{1}=0$, say. Then $\mu_{0}=0$. Putting $x_{2: n}=\underline{1}_{n-1}$, we find $E(\underline{x})=\sum_{i=2}^{n} b_{i}-\mu x_{1}^{a_{1}}$. Going with $x_{1}$ towards $-\infty$ or $+\infty$ we see that psd-ness of $E$ implies $\mu \leq 0$ and $a_{1}$ is even. If there would be an odd $a_{i}$ then choosing $x_{i}=-1$ (maintaining the other $x_{2}, \ldots, x_{n}$ equal to 1 ) yields $E(\underline{x})=\sum_{i=2}^{n} b_{i}+\mu x_{1}^{a_{1}}$. Then $\mu<0$ again yields a contradiction. So either $\mu<0$ and all $a_{i}$ are even or $\mu=0$. This shows ii in the case that some $b_{i}=0$.

Assume now that $b_{i}>0$ for $i=1, \ldots, n$. Then the map

$$
\mathbb{R}_{\geq 0}^{n} \ni\left(u_{1}, u_{2}, \ldots, u_{n}\right) \stackrel{T}{\mapsto}\left(\left(\frac{a_{1}}{2 d b_{1}} u_{1}\right)^{1 / 2 d}, \ldots,\left(\frac{a_{n}}{2 d b_{n}} u_{n}\right)^{1 / 2 d}\right) \in \mathbb{R}_{\geq 0}^{n}
$$


is well defined. It is easy to see that $T$ is a bijectve. Now

$$
E(T(x))=\frac{a_{1}}{2 d} x_{1}+\ldots+\frac{a_{n}}{2 d} x_{n}-\underbrace{\left(\frac{\mu}{2 d} \prod_{i=1}^{n}\left(\frac{a_{i}}{b_{i}}\right)^{a_{i} / 2 d}\right)}_{\mu_{1}} \cdot \prod_{i=1}^{n} x_{i}^{a_{i} / 2 d} .
$$

By the arithmetic-geometric mean inequality, see e.g. [BB, p13c-0], and definition of $\mu_{1}$, we thus find $E \mid \mathbb{R}_{\geq 0}^{n} \geq 0$ iff $E \circ T \mid \mathbb{R}_{\geq 0}^{n} \geq 0$ iff $\mu_{1} \leq 1$ iff $\mu \leq \mu_{0}$. Evidently we also have

$E \mid \mathbb{R}^{n} \geq 0$ iff for all $\underline{\varepsilon} \in\{-1,+1\}^{n}$ and $\underline{x} \in \mathbb{R}_{\geq 0}^{n} E_{\underline{\varepsilon}}(\underline{x})=E(\underline{\varepsilon} \circ \underline{x}) \geq 0$, where $\underline{\varepsilon} \circ \underline{x}=\left(\varepsilon_{1} x_{1}, \ldots, \varepsilon_{n} x_{n}\right)$.

Now $E_{\underline{\varepsilon}}(\underline{x})=E(\underline{\varepsilon} \circ \underline{x})=D(\underline{x})-\mu \prod_{i=1}^{n} \varepsilon_{i}^{a_{i}} \underline{x}^{\underline{a}}$. So what we found above is applicable to $E_{\underline{\varepsilon}}$, that is, $E_{\underline{\varepsilon}} \mid \mathbb{R}_{\geq 0}^{n} \geq 0$, iff $\mu \prod_{i=1} \varepsilon_{i}^{a_{i}} \leq \mu_{0}$. This will hold for every $\underline{\varepsilon} \in\{-1,+1\}^{n}$ iff (ii) holds. This establishes (ii).

$\mathrm{i} \Leftarrow \mathrm{ii}$. Assume $b_{i}=0$ for some $i$. Then $\mu_{0}=0$ and (ii) implies by virtue of $\mu \neq 0$ that $\mu<0$ and all $a_{i}$ are even. This implies obviously that $E$ is sobs and hence psd. In the case that all $b_{i}>0$, the equivalences we have used in the implication before yield (i).

ii $\Rightarrow$ iii. Assume (ii). We have observed just before that existence of some $b_{i}=0$ implies $E$ is sos. So we assume now all $b_{i}>0$. Then we have a well defined bijective map from $\mathbb{R}^{n}$ to $\mathbb{R}^{n}$ given by

$$
\mathbb{R}^{n} \ni\left(u_{1}, u_{2}, \ldots, u_{n}\right) \stackrel{T}{\mapsto}\left(\left(\frac{a_{1}}{2 d b_{1}}\right)^{1 / 2 d} u_{1}, \ldots,\left(\frac{a_{n}}{2 d b_{n}}\right)^{1 / 2 d} u_{n}\right) \in \mathbb{R}^{n} .
$$

Then

$$
G(\underline{x})=2 d E(T(\underline{x}))=a_{1} x_{1}^{2 d}+\ldots+a_{n} x_{n}^{2 d}-\mu_{1} 2 d \cdot \prod_{i=1}^{n} x_{i}^{a_{i}} .
$$

Since $T$ is bijective the equivalence of (i) and (ii) already proved and applied to $G(\underline{x})$ yields $E(\underline{x})$ is psd iff $G(\underline{x})$ is psd iff $\mu_{1} \leq 1$, and all $a_{i}$ are even or $\left|\mu_{1}\right| \leq 1$ and some $a_{i}$ are odd.

If $\mu_{1}<-1$, then all the $a_{i}$ are even; and since $-\mu_{1} \cdot 2 d>0$, it is clear that $G(\underline{x})$ is sum of $n+1$ monomial squares. 
Now assume $0 \leq \mu_{1} \leq 1$. We can write

$$
G(\underline{x})=2 d \mu_{1}\left(\frac{a_{1}}{2 d} x_{1}^{2 d}+\ldots+\frac{a_{n}}{2 d} x_{n}^{2 d}-\prod_{i=1}^{n} x_{i}^{a_{i}}\right)+2 d\left(1-\mu_{1}\right)\left(\sum_{i=1}^{n} \frac{a_{i}}{2 d} x_{i}^{2 d}\right)
$$

Noting that $\left(a_{1}, \ldots, a_{n}\right)=\sum_{i=1}^{n} \frac{a_{i}}{2 d} 2 d \underline{e}_{i}$, we see that the expression in the first pair of parentheses is a simplicial agiform on a $H$-trellis, and therefore by lemma 2.2 a sum of binomial squares. Hence evidently $G(\underline{x})$ is a sum of binomial and monomial squares.

Finally assume $-1 \leq \mu_{1}<0$. If all $a_{i}$ are even then $G(\underline{x})$ is a sum of monomial squares. So assume now some of the $a_{i}$, among others, w.l.o.g. $a_{1}$, say, are odd. Then since $\left|\mu_{1}\right| \leq 1$, we have by the result just proved that

$$
\tilde{G}(\underline{x})=G\left(-x_{1}, x_{2: n}\right)=a_{1} x_{1}^{2 d}+\ldots+a_{n} x_{n}^{2 d}-\left|\mu_{1}\right| 2 d \cdot \prod_{i=1}^{n} x_{i}^{a_{i}}
$$

is a sum of binomial and monomial squares. Since the property of being a square of a binomial or monomial remains invariant under change of sign of $x_{1}$, it follows that $G(\underline{x})=G\left(x_{1}, x_{2: n}\right)=\tilde{G}\left(-x_{1}, x_{2: n}\right)$ is a sum of binomial and monomial squares. Finally, since $E(\underline{x})=\frac{1}{2 d} G\left(T^{-1}(\underline{x})\right)$, and since the linear diagonal operator $T^{-1}$ transforms binomials into bionomials, (iii) follows.

$\mathrm{iii} \Rightarrow \mathrm{i}$. This implication is trivial.

b. In the implication $\mathrm{i} \Rightarrow$ ii above we have established using the arithmetic geometric inequality that $E(T(x)) \geq 0$. The sharper form of the inequality tells us also that in case $\mu_{1}=1$ we have for $\underline{x} \in \mathbb{R}_{\geq 0}^{n}, \underline{x} \neq \underline{0}$, that $E(T(\underline{x}))=0$ iff $x_{1}=\ldots=x_{n}$; that is iff $x_{1}: \ldots: x_{n}=1: 1: \ldots: 1$. Now $T\left(\underline{1}_{n}\right)=$ $\left(\left(\frac{a_{1}}{2 d b_{1}}\right)^{1 / 2 d}, \ldots,\left(\frac{a_{n}}{2 d b_{n}}\right)^{1 / 2 d}\right)$, and since $T$ is bijective on $\mathbb{R}_{\geq 0}^{n}$, the claim follows.

To fix notation for the sequel, we consider a dmt form $F$ written as

$$
F(\underline{x})=F\left(x_{1}, \ldots, x_{n}\right)=\sum_{i=1}^{n} b_{i} x_{i}^{2 d}-\sum_{\underline{i} \in I} a_{\underline{i}} \underline{x}^{\underline{i}},
$$

with

$$
I=\left\{\underline{i} \in \mathbb{Z}_{\geq 0}^{n}: a_{\underline{i}}>0, \text { all } i_{\nu} \leq 2 d-1\right\}
$$


The standard $n-1$-simplex in the hyperplane $H_{n-1}=\left\{\underline{x} \in \mathbb{R}^{n}: \sum_{i} x_{i}=1\right\}$ is $\Delta_{n-1}=H_{n-1} \cap \mathbb{R}_{\geq 0}^{n}$. Its (relative) interior and its (relative) boundary are $\operatorname{int}\left(\Delta_{n-1}\right)=\left\{\underline{x} \in \Delta_{n-1}: \forall i x_{i}>0\right\}$, and bd $\left(\Delta_{n-1}\right)=\left\{\underline{x} \in \Delta_{n-1}: \exists i x_{i}=0\right\}$, respectively.

The following lemma will be generalized in corollary 2 .

Lemma 2.4. Let $F$ be a indecomposable psd dmt form $F$ of dimension $n \geq 2$. Then $F \mid \Delta_{n-1}$ has a local minimum in int $\left(\Delta_{n-1}\right)$.

Proof. The first case to consider is $\operatorname{dim}(F)=2$. Here we have to consider a form

$$
F(x, y)=b_{1} x^{2 d}+b_{2} y^{2 d}-\sum_{\substack{i \geq 1, j \geq 1 \\ i+j=2 d}} a_{i j} x^{i} y^{j}
$$

on $\Delta_{1}=\{(x, y): x+y=1, x, y \geq 0\}$. By lemma 1a, $\operatorname{var}(D)=\{x, y\}$ since otherwise the dimension would be 1 . So $b_{1}, b_{2}>0$. Note $\operatorname{bd}\left(\Delta_{1}\right)=$ $\{(1,0),(0,1)\}$ and $F(1,0)=b_{1}$ and $F(0,1)=b_{2}$. The natural derivative $D$ on the line $x+y=1$ is given by

$$
D F(x, y):=\left.\frac{d F}{d t}(x-t, y+t)\right|_{t=0}=\left(\frac{\partial f}{\partial y}-\frac{\partial f}{\partial x}\right)(x, y) .
$$

Since $D F(1,0)=-2 d b_{1}-a_{2 d-1,1}<0$ and similarly $D F(0,1)=2 d b_{2}+$ $a_{1,2 d-1}>0$, we find that at points $(x, y) \in$ int $\left(\Delta_{1}\right)$ in the neighborhood of $(1,0)$ and $(0,1)$ we have $F(x, y)<F(1,0)$ and $F(x, y)<F(0,1)$, respectively. Consequently we have a local minimum in int $\left(\Delta_{1}\right)$. Note that this argument works hypothizing $b_{1}, b_{2}>0$, even without indecomposability.

We return to the general case. Let $\Delta=\Delta_{n-1}$. Assume there exists $\underline{z} \in \Delta$ so that $F(\underline{z})=0$. Assume $\left\{i: z_{i}=0\right\} \neq \emptyset$; w.l.o.g. $\left\{i: z_{i}=0\right\}=\{1,2, \ldots, k\}$, say. Then $\underline{z}=\left(0, \ldots, 0, z_{k+1}, \ldots, z_{n}\right)$. By lemma 2.1 a the variables $x_{1}, \ldots, x_{n}$ occur in $D$. So $D(\underline{z})>0$, consequently $T(\underline{z})>0$. So, there occur terms $a_{\underline{i}} \underline{z}^{\underline{i}}=a_{\underline{i}} 0^{i_{1}} \ldots 0^{i_{k}} z_{k+1}^{i_{k+1}} \cdots z_{n}^{i_{n}} \neq 0$ in $T(\underline{z})$, and we must conclude $i_{1}=\ldots=i_{k}=$ 0 for all such terms. Thus $x_{1}, \ldots, x_{k} \notin \operatorname{var}(T)$. But since $x_{1}, \ldots, x_{k} \in \operatorname{var}(D)$, we find that $F$ decomposes as $F=\left(\sum_{i=1}^{k} b_{i} x_{i}^{2 d}\right)+\left(\sum_{i=k+1}^{n} b_{i} x_{i}^{2 d}-T(\underline{x})\right)$, a contradiction. This shows: a zero $\underline{z}$ of $F \mid \Delta_{n-1}$ if existing must lie in int $\left(\Delta_{n-1}\right)$. Hence psd-ness of $F$ implies that for all $\underline{x} \in$ bd $(\Delta)$ we have $F(\underline{x})>0$. 
Since $\operatorname{bd}(\Delta)$ is compact we infer that there exists a point $\underline{\hat{x}} \in \mathrm{bd}(\Delta)$ such that $0<F(\underline{\hat{x}})=\min F \mid$ bd $(\Delta)$. Assume w.l.o.g. that $\underline{\hat{x}}=\left(\hat{x}_{1}, \ldots, \hat{x}_{n-1}, 0\right)$.

For any pair $x, y$ with $x+y=1$, we compute

$\tilde{F}(x, y)=F\left(x \underline{\hat{x}}+y \underline{e}_{n}\right)=\left(\sum_{i=1}^{n-1} b_{i} \hat{x}_{i}^{2 d}\right) x^{2 d}+b_{n} y^{2 d}-\sum_{\underline{i} \in I} a_{\underline{i}} \hat{x}_{1}^{i_{1}} \ldots \hat{x}_{n-1}^{i_{n-1}} x^{i_{1}+\ldots+i_{n-1}} y^{i_{n}}$.

Note $\tilde{F}(1,0)=F(\underline{\hat{x}})>0$. Also $\underline{e}_{n} \in$ bd $(\Delta)$ guarantees $\tilde{F}(0,1)=F\left(\underline{e}_{n}\right) \geq$ $F(\underline{\hat{x}})>0$. This guarantees by the case $\operatorname{dim}(F)=2$ considered at the beginning that there are $0<\bar{x}, \bar{y}<1, \bar{x}+\bar{y}=1$ so that $\tilde{F}(\bar{x}, \bar{y})<\tilde{F}(1,0)=F(\underline{\hat{x}})$. The point $\bar{x} \underline{\hat{x}}+\bar{y} \underline{e}_{n}$ is not a boundary point of $\Delta$, for otherwise we would have a contradiction to the choice of $\underline{\hat{x}}$. Hence this point is in the relative interior of $\Delta$ and at it $F$ assumes a smaller value than at any point of bd $(\Delta)$. So $F \mid \Delta$ has a global, hence local minimum in $\operatorname{int}(\Delta)$.

Deleting the word 'indecomposable' the previous lemma becomes false. The decomposable psd dmt form $x^{4}+y^{4}+z^{4}-2 x^{2} y^{2}=\left(x^{2}-y^{2}\right)^{2}+z^{4}$ on $\Delta_{2}$ has its only local minimum at $(x, y, z)=(.5, .5,0) \in \mathrm{bd}\left(\Delta_{2}\right)$

Next we show how to construct elementary positive semi definite dmt-forms of prescribed tail and entrywise positive zero.

Proposition 2.5. Let $\underline{u} \in \operatorname{int}\left(\Delta_{n-1}\right), a_{1}, \ldots, a_{n} \in \mathbb{Z}_{\geq 0}, \sum_{i=1}^{n} a_{i}=2 d \geq 2$, and $\mu>0$. Define $b_{i}, i=1, \ldots, n$ and a form $E$ by

$$
b_{i}=\frac{\mu}{2 d} a_{i} \frac{u_{1}^{a_{1}} \cdots u_{n}^{a_{n}}}{u_{i}^{2 d}} \text { and } E(\underline{x})=\sum_{i=1}^{n} b_{i} x_{i}^{2 d}-\mu x_{1}^{a_{1}} \cdots x_{n}^{a_{n}} .
$$

Then the $b_{i}$ are well defined. If $E \neq 0$ then $E$ is a indecomposable psd dmt form and has a zero is $\underline{u}$. If $\operatorname{dim}(E)=n, \underline{u}$ is the unique zero in $\Delta_{n-1}$.

Proof. The hypotheses on $\underline{u}$ and $d$ imply that $b_{i}$ is well defined. Furthermore we have $a_{i}=0$ iff $b_{i}=0$ from where it follows that $E$ is either indecomposable or identically 0 (which is the case iff $a_{i}=2 d$ for some $i$ ). Assume now $E \neq 0$. If $\operatorname{dim}(E)=n$, we can compute the threshold value $\mu_{0}$ by means of theorem 
2.3. With the current definitions of the $b_{i}$, we find

$$
\begin{aligned}
\mu_{0} & =2 d \prod_{i=1}^{n}\left(\frac{b_{i}}{a_{i}}\right)^{a_{i} / 2 d} \\
& =2 d \prod_{i=1}^{n}\left(\frac{\mu}{2 d} \frac{u_{1}^{a_{1}} \cdots u_{n}^{a_{n}}}{u_{i}^{2 d}}\right)^{a_{i} / 2 d} \\
& =\mu \prod_{i=1}^{n} \frac{\left(u_{1}^{a_{1}} \cdots u_{n}^{a_{n}}\right)^{a_{i} / 2 d}}{u_{i}^{a_{i}}} \\
& =\mu,
\end{aligned}
$$

since $\sum a_{i}=2 d$. So by theorem $2.3 E$ is psd and has in $\Delta_{n-1} \subseteq \mathbb{R}_{\geq 0}^{n}$ only one zero.

If $\operatorname{dim}(E) \leq n-1$, then some of the variables $x_{i}$ do not occur; i.e. the set $I=\left\{i: a_{i}=0\right\}=\left\{i: b_{i}=0\right\}$ is nonempty. Let $\Delta^{\prime}=\left\{\underline{x} \in \Delta: x_{i}=\right.$ 0 , for $i \in I\} . \Delta^{\prime}$ is an $n-|I|-1$-dimensional simplex and we can repeat above calculations and reasoning for $E(\underline{x})$ restricted to $\Delta^{\prime}$. The indices $i$ above have to be restricted to the $i \in I^{c}$. We get that $\underline{u}^{\prime}$ defined by $u_{i}^{\prime}=u_{i}$ iff $i \in I^{c}$ and $u_{i}^{\prime}=0$ iff $i \in I$ is a zero of $E$. But since $E$ does not depend of the $x_{i}, i \in I, \underline{u}$ is also a zero of $E$ and we are done.

Lemma 2.6. Let $F$ be a psd homogeneous polynomial of positive degree and dimension $n$. Assume $\underline{u} \in \operatorname{int}\left(\Delta_{n-1}\right)$ is a local minimum of $F \mid \Delta_{n-1}$. Then there exists a $\lambda \leq 0$ such that $0=\nabla F(\underline{u})+\lambda \underline{1}_{n}$.

Proof. Consider the function $[0, \infty[\ni t \stackrel{\varphi}{\mapsto} F(t \underline{u})$. By our homogeneity assumptions, $\varphi(t)=t^{d} F(\underline{u})$ for some $d \geq 1$. Since $\underline{u} \in \Delta, I=\left\{i: u_{i} \neq 0\right\}=$ $\left\{i: u_{i}>0\right\} \neq \emptyset$. Hence $F(\underline{u}) \geq 0$ implies

$$
0 \leq \frac{d \varphi(t)}{d t}=\frac{d}{d t} F(t \underline{u})=\sum_{i=1}^{n} \frac{\partial F}{\partial x_{i}}(t \underline{u}) u_{i}=\sum_{i \in I} \frac{\partial F}{\partial x_{i}}(t \underline{u}) u_{i} .
$$

So there exists an $i \in I$ such that $\frac{\partial F}{\partial x_{i}}(\underline{u}) \geq 0$.

We have $\nabla\left(\sum_{i=1}^{n} x_{i}\right)=\underline{1}_{n}$. So the Lagrange Caratheodory multiplier rule (see $[\mathrm{Po}]$ for a geometric discussion not often found in textbooks) tells us that there is a $\lambda$ so that $0=\nabla F(\underline{u})+\lambda \underline{1}_{n}$. In particular for the chosen $i \in I$, $\frac{\partial F}{\partial x_{i}}(\underline{u})+\lambda=0$, implying $\lambda \leq 0$. 
Theorem 2.7. Let $F$ be a psd dmt form. Then $F$ is a sum of elementary psd dmt forms and in particular sum of binomial squares.

Proof. Assume first that $F$ is an indecomposable form of dimension $n$. Let $\Delta=\Delta_{n-1}$.

By lemma 2.4 we know that there exists a local minimum $\underline{u}$ of $F$ in int $(\Delta)$. Using notation (2.3'), by lemma 2.6 we have for some $\lambda \leq 0$ the equations

$$
2 d b_{k} u_{k}^{2 d-1}-\sum_{\underline{i} \in I} a_{\underline{i}} \cdot i_{k} \cdot u_{1}^{i_{1}} \ldots u_{k}^{i_{k}-1} \ldots u_{n}^{i_{n}}+\lambda=0, \quad k=1, \ldots, n .
$$

Putting

$$
b_{\underline{i k}}:=\frac{a_{\underline{i}}}{2 d} i_{k} \frac{\underline{u^{\underline{i}}}}{u_{k}^{2 d}}
$$

we find for $k=1, \ldots, n$, that

$$
\begin{aligned}
b_{k} & =\sum_{\underline{i} \in I} \frac{a_{\underline{i}}}{2 d} i_{k} \frac{u_{1}^{i_{1}} \ldots u_{k}^{i_{k}-1} \ldots u_{n}^{i_{n}}}{u_{k}^{2 d-1}}-\frac{\lambda}{2 d u_{k}^{2 d-1}} \\
& =\sum_{\underline{i} \in I} b_{\underline{i} k}-\frac{\lambda}{2 d u_{k}^{2 d-1}} .
\end{aligned}
$$

For each $\underline{i} \in I$, proposition 2.5 tells us that

$$
E_{\underline{i}}(\underline{x})=\sum_{k=1}^{n} b_{\underline{i} k} x_{k}^{2 d}-a_{\underline{i}} \underline{x}^{\underline{i}}
$$

is a elementary indecomposable psd dmt form that has a zero at $\underline{u}$. Now we compute

$$
\begin{aligned}
F(\underline{x}) & =\sum_{k=1}^{n} b_{k} x_{k}^{2 d}-\sum_{\underline{i} \in I} a_{\underline{i}} \underline{x}^{\underline{i}} \\
& =\sum_{k=1}^{n}\left(\sum_{\underline{i} \in I} b_{\underline{i} k}-\frac{\lambda}{2 d u_{k}^{2 d-1}}\right) x_{k}^{2 d}-\sum_{\underline{i} \in I} a_{\underline{i}} \underline{x}^{\underline{i}} \\
& =\sum_{\underline{i} \in I}\left(\sum_{k=1}^{n} b_{\underline{i} k} x_{k}^{2 d}-a_{\underline{i}} \underline{x}^{\underline{i}}\right)-\sum_{k=1}^{n} \frac{\lambda}{2 d u_{k}^{2 d-1}} x_{k}^{2 d} \\
& =\sum_{\underline{i} \in I} E_{\underline{i}}(\underline{x})+\sum_{k=1}^{n} \frac{(-\lambda)}{2 d u_{k}^{2 d-1}} x_{k}^{2 d}
\end{aligned}
$$


Since $-\lambda \geq 0$, the last summand is a elementary psd dmt form without tail. We thus have shown that every indecomposable psd dmt form is a sum of elementary psd dmt forms. In the general case, by lemma $2.1 \mathrm{~b}$, one can write $F=F_{1}+F_{2}+\ldots+F_{l}$ with indecomposable psd dmt forms. Write each of these as a sum of elementary psd dmt forms. Evidently, each elementary psd dmt form $E=E^{\prime}+D^{\prime}$ with an indecomposable elementary psd dmt form $E^{\prime}$ and a psd diagonal form $D^{\prime}$. By theorem 2.3, $E^{\prime}$ is sobs and obviously $D^{\prime}$ is sum of monomial squares. The theorem follows.

Originally we tried to establish the uniqueness result of the following corollary as a base for theorem 2.7. An attempt for a direct proof caused us considerable troubles even in the case $n=2$. It is amusing that rather than a base, it is an easy consequence of theorem 2.7 .

Corollary 2.8. (of proof of theorem 2.7) Let $F$ be a indecomposable psd dmt form of dimension $n$. Then $F$ has on $\Delta_{n-1}$ a unique local minimum; it is of course also the global minimum of $F \mid \Delta_{n-1}$.

Proof. In the proof of theorem 2.7 we established that if $\underline{u}$ is a by lemma 2.4 existing local minimum of $F \mid \Delta_{n-1}$, then we can write

$$
F(\underline{x})=\sum_{\underline{i} \in I} E_{\underline{i}}(\underline{x})+D^{\prime}(\underline{x}) \text {, with diagonal } D^{\prime}(x)=\sum_{k=1}^{n} \frac{(-\lambda)}{2 d u_{k}^{2 d-1}} x_{k}^{2 d}
$$

for certain elementary indecomposable psd dmt forms $E_{i}(\underline{x}), \underline{i} \in I$ which all have $\underline{u}$ as a zero in $\Delta_{n-1}$. The zero is unique for all forms $E_{\underline{i}}$ of dimension $n$. However in general only the components $u_{i}$ for which $x_{i} \in \operatorname{var}\left(E_{\underline{i}}\right)$ are unique, the other components are arbitrary. For any $\underline{x} \in \Delta_{n-1}$ and any $\underline{i} \in I$ we have $*: E_{i}(\underline{x}) \geq 0=E_{i}(\underline{u})$. Now since $F$ is indecomposable of dimension $n$ we find that $\operatorname{var}(F)=\bigcup_{\underline{i} \in I} \operatorname{var}\left(E_{\underline{i}}\right)$. Thus for any $\underline{x}$ the inequality $*$ is strict for some $\underline{i}$.

Viewing the $E_{i}, F$, and $D^{\prime}$ as functions on the hyperplane $H_{n-1}$ containing $\Delta_{n-1}$, we now see that $\sum_{\underline{i} \in I} E_{\underline{i}}(\underline{x})>\sum_{\underline{i} \in I} E_{\underline{i}}(\underline{u})=0$ for all $\underline{x} \in \Delta_{n-1} \backslash\{\underline{u}\}$. Further, by Lagrange multipliers applied to $D^{\prime} \mid H_{n-1}$ we see that provided $D^{\prime} \neq 0, D^{\prime} \mid H_{n-1}$ has only one local (and hence global) minimum, namely in $\underline{u}$. Consequently $D^{\prime}(\underline{x})>D^{\prime}(\underline{u})$ for all $\underline{x} \in H_{n-1} \backslash\{\underline{u}\}$. This proves: for all $\underline{x} \in \Delta_{n-1} \backslash\{\underline{u}\}$ we have $F(\underline{x})>F(\underline{u})$. It follows that $F \mid \Delta_{n-1}$ has $\underline{u}$ as its unique global minimum. Since our reasoning began with an arbitrary 
local minimum and showed it is the unique global minimum, the claim is established.

Sometimes one will be able to show that a given form is sobs by relating it to a psd dmt form via the following corollary.

Corollary 2.9. (of proof of theorem 2.7) Consider the forms $P$ and $F$ of degree $2 d$ given by

$$
P(\underline{x})=\sum_{i=1}^{n} b_{i} x_{i}^{2 d}+\sum_{\underline{i} \in I} a_{\underline{i}} \underline{x}^{\underline{i}} \text { and } F(\underline{x})=\sum_{i=1}^{n} b_{i} x_{i}^{2 d}-\sum_{\underline{i} \in I}\left|a_{\underline{i}}\right| \underline{x}^{\underline{i}},
$$

where for all $\underline{i} \in I$, $\max \underline{i} \leq 2 d-1$. If $F(\underline{x})$ is psd, then $P(\underline{x})$ is sum of squares of binomials.

Proof. The conditions imposed on the $\underline{i}$ guarantee that $F(\underline{x})$ is a dmt form. Evidently there exist signs $\varepsilon_{\underline{i}} \in\{-1,+1\}$ such that

$$
P(\underline{x})=\sum_{i=1}^{n} b_{i} x_{i}^{2 d}-\sum_{\underline{i} \in I} \varepsilon_{\underline{i}}\left|a_{\underline{i}}\right| \underline{x}^{\underline{i}} .
$$

Let us assume first and for simplicity of notation that $F=D-T$ is indecomposable, where $D$ and $T$ are diagonal and tail of $F$, respectively.

From the proof of theorem 2.7 we know that $F(\underline{x})=\sum_{\underline{i} \in I} E_{\underline{i}}(\underline{x})+D^{\prime}(\underline{x})$ for certain elementary indecomposable psd dmt forms $E_{\underline{i}}(\underline{x})=D_{\underline{i}}(\underline{x})-\left|a_{\underline{i}}\right| \underline{x}^{\underline{i}}$, where $D_{i}, D^{\prime}$ are psd diagonal and hence sobs. To each $E_{i}$ associate $E_{\underline{i}}^{\prime}(\underline{x})=D_{\underline{i}}(\underline{x})-\varepsilon_{\underline{i}}\left|a_{\underline{i}}\right| \underline{x}^{\underline{i}}$. By theorem $2.3 \mathrm{a} E_{\underline{i}}^{\prime}$ is psd and hence sobs. Evidently $P(\underline{x})=\sum_{\underline{i} \in I} E_{\underline{i}}^{\prime}(\underline{x})+D^{\prime}(\underline{x})$. Thus $P$ is sobs.

In the general case of a decomposable form $F$, by lemma 1 b we can write $F=F_{1}+\ldots+F_{k}$ as a sum of indecomposable psd dmt forms $F_{i}=D_{i}-T_{i}$, $i=1, \ldots, k$, where $D_{i}$ and $T_{i}$ are diagonal and tail of $F_{i}$ respectively and can apply above reasoning to each of the indecomposable forms $F_{i}$. Invoking lemma 1c concludes the proof that $P$ is sobs.

Robinson [Ro, p269] has shown the following interesting fact by a quite different method.

Corollary 2.10. If $F(\underline{x})$ is any real form of degree 2 d, then for large enough $\beta$, the polynomial $P(\underline{x})=F(\underline{x})+\beta\left(x_{1}^{2 d}+\ldots x_{n}^{2 d}\right)$ is a sum of squares of binomials. 
Proof. Change all positive coefficients in $F(\underline{x})$ not pertaining to a monomials $x_{i}^{2 d}$ to their negative counterpart. Let $\hat{F}(\underline{x})$ be the resulting polynomial. On the unit sphere it assumes its minimum. Hence for large enough $\beta$, the polynomial $\hat{F}(\underline{x})+\beta\left(x_{1}^{2 d}+\ldots x_{n}^{2 d}\right)$ will be a positive definite diagonal minus tail form and hence sobs by theorem 2.7. Since the operation 'adding $\beta \sum_{i} x_{i}^{2 d}$ ' and 'changing signs of monomials $\neq x_{i}^{2 d}$ ' commute, corollary 2.9 guarantees that $P(\underline{x})$ will be sobs.

\section{An Algorithm and Examples}

The theorems of this paper are constructive. To decide whether a diagonal minus tail form $F(\underline{x})$ can be written as a sum of squares and if so to give the an explicit representation, one can use the following algorithm.

\section{Algorithm 3.1.}

1. Write the complete decomposition of $F, F=F_{1}+\ldots+F_{k}$ into dmt forms $F_{i}$; see lemma 2.1.

2. Determine for each of the indecomposable $F_{i}$ the by corollary 2.8 unique local minimum $\underline{u}_{i}$ in the relative interior of the standard simplex spanned by the $\underline{e}_{\ell}$ for which $x_{\ell} \in \operatorname{var}\left(F_{i}\right)$.

3. If there exists an $i$ such that $F_{i}\left(\underline{u}_{i}\right)<0$, then stop: $F_{i}$ and hence by lemma $2.1 \mathrm{~b} F$ cannot be sum of squares.

4. Otherwise write each of the $F_{i}$ as a sum of elementary nondiagonal dmt forms plus one diagonal form as in the proof of theorem 2.7 (which recurs to proposition 2.5).

5. Write each of the nondiagonal elementary dmt forms obtained in step 4 according to the proof of theorem $2.3 \mathrm{a}, \mathrm{ii} \Rightarrow \mathrm{iii}$ as a sum of squares of binomials. This also requires applying the method given in the proof of [Re2], theorem 4.4 to $\mathcal{L}=C\left(\mathcal{H}_{n, 2 d}\right)$.

6. Upon summing the sos representations of all the $F_{i}$ in step 5 and the respective diagonal forms of step 4 we get a representation of $F$ as a sum of binomial and monomial squares.

To illustrate the algorithm we consider the example

$$
F(x, y, z)=x^{4}+y^{4}+z^{4}+2 v^{4}+w^{4}-0.7 x^{3} y-0.9 x^{2} y z-y z^{3}-v^{2} w^{2} .
$$


This is a dmt form. As step 1 we note that the complete decomposition of $F$ is $F=F_{1}+F_{2}$ with

$$
F_{1}=x^{4}+y^{4}+z^{4}-0.7 x^{3} y-0.9 x^{2} y z-y z^{3} \text {, and } F_{2}=2 v^{4}+w^{4}-v^{2} w^{2} \text {. }
$$

We next find that the local minimum of $F_{1}$ on $\Delta_{2}$ is assumed in $\underline{u}_{1}=$ $(0.343,0.313,0.343)$. Here and below we cut off the digits following the third one; much more precise values are listed below to 16 decimal places. Since $F_{1}\left(\underline{u}_{1}\right)=0.004>0, F_{1}$ is positive semidefinite. Similarly $F_{2}$ assumes on $\Delta_{1}$ its local minimum in $\underline{u}_{2}=(0.448,0.551)$ and the value of $F_{2}\left(\underline{u}_{2}\right)=0.112>0$. This completes steps 2,3 of the algorithm.

Next we have to write $F_{1}$ and $F_{2}$ as sums of elementary dmt forms. By the proof of theorem 2.7 we can find for $F_{1}$ a representation $F_{1}=E_{11}+E_{12}+$ $E_{13}+D_{1}$, where $D_{1}$ is psd diagonal, and the $E_{1 i}$ are elementary dmt forms having respective tails $-0.7 x^{3} y,-0.9 x^{2} y z,-1 y z^{3}$, and the common zero $\underline{u}_{1}$; for $F_{2}$ we have similarly to find a representation $F_{2}=E_{21}+D_{2}$ where $E_{21}$ has tail $-v^{2} w^{2}$ and zero $\underline{u}_{2}$, and $D_{2}$ is diagonal.

Proposition 2.5 gives us formulae for finding the $E$ s. The $D$ s are then found by the obvious subtractions. The result of these calculations are as follows. For $F_{1}$,

$$
\begin{aligned}
E_{11} & =0.479 x^{4}+0.230 y^{4}+0 z^{4}-.7 x^{3} y, \\
E_{12} & =0.410 x^{4}+0.295 y^{4}+0.205 z^{4}-.9 x^{2} y z \\
E_{13} & =0 x^{4}+0.328 y^{4}+0.684 z^{4}-1.0 y z^{3} \\
D_{1} & =0.110 x^{4}+0.144 y^{4}+0.110 z^{4}
\end{aligned}
$$

while for $F_{2}$,

$$
\begin{aligned}
E_{21} & =0.755 v^{4}+0.330 w^{4}-v^{2} w^{2} \\
D_{2} & =1.244 v^{4}+0.669 w^{4} .
\end{aligned}
$$

This was step 4 . Next for step 5, glancing at the proof of theorem 2.3 we find that if $E$ is a elementary dmt form with a zero, then there $\mu_{1}=1$. We shall apply the formula

$$
G(\underline{x})=2 d E(T(x))=2 d\left(\frac{a_{1}}{2 d} x_{1}^{2 d}+\ldots+\frac{a_{n}}{2 d} x_{n}^{2 d}-\prod_{i=1}^{n} x_{i}^{a_{i}}\right)
$$


with $d=2$ and $n=3$ in case of $E=E_{12}$ and $n=2$ for $E_{11}, E_{13}, E_{21}$, write $G(\underline{x})$ as sobs using Reznick's agiform theory, and obtain the desired sobs representations for $E$ as $E(\underline{x})=\frac{1}{4} G\left(T^{-1}(\underline{x})\right)$.

We do the case $E=E_{12}$ in detail, and let the remaining simpler cases for verification to the reader.

With $\underline{\lambda}=\left(\frac{a_{1}}{2 d}, \ldots, \frac{a_{n}}{2 d}\right), \underline{w}=\left(a_{1}, \ldots, a_{n}\right)$, the form in parenthesis is in Reznick's notation the simplicial agiform $f\left(\mathcal{H}_{n, 2 d}, \underline{w}\right)=f\left(\mathcal{H}_{n, 2 d}, \underline{\lambda}, \underline{w}\right)=\frac{G}{2 d}$. For $E=E_{12}$, the relevant objects are $\underline{w}=(2,1,1)$, and

$$
\mathcal{U}^{*}=H_{3,4}^{*}=C\left(\mathcal{H}_{3,4}\right)=\operatorname{cvx}\{(0,0,4),(0,4,0),(4,0,0)\} .
$$

Since $\mathcal{H}_{3,4}$ is by p438c- 4 a trellis, $\mathcal{F}=E\left(\mathcal{H}_{3,4}\right) \cup\{\underline{w}\}$ is by theorem 2.8, p440 a $\mathcal{U}$-mediated set. Then the proof of theorem 4.4, p443 tells us how to write $f\left(\mathcal{H}_{3,4},(2,1,1)\right)$ as a sobs. Namely, with the notations in our figure, reminiscent of the figure on $\mathrm{p} 435$, we find that $\underline{w}_{1}, \ldots, \underline{w}_{4}$ satisfies in terms of Reznick's case distinctions (4.5) the following cases, respectively.

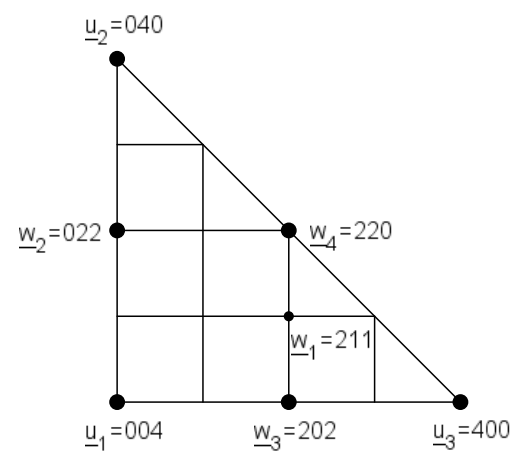

$$
\begin{array}{ll}
\underline{w}_{1}=\frac{1}{2}\left(w_{3}+w_{4}\right) & \text { case } \text { iii }, \\
\underline{w}_{2}=\frac{1}{2}\left(u_{1}+u_{2}\right) & \text { case i }, \\
\underline{w}_{3}=\frac{1}{2}\left(u_{1}+u_{3}\right) & \text { case i }, \\
\underline{w}_{4}=\frac{1}{2}\left(u_{2}+u_{3}\right) & \text { case i . }
\end{array}
$$

Hence we have to invert the matrix

$$
A=\left[\begin{array}{cccc}
2 & 0 & -1 & -1 \\
0 & 2 & 0 & 0 \\
0 & 0 & 2 & 0 \\
0 & 0 & 0 & 2
\end{array}\right], \text { obtaining } 4 A^{-1}=\left[\begin{array}{cccc}
2 & 0 & 1 & 1 \\
0 & 2 & 0 & 0 \\
0 & 0 & 2 & 0 \\
0 & 0 & 0 & 2
\end{array}\right]
$$


So by the reasoning in theorem $4.4,4 f\left(\mathcal{H}_{3,4}, \underline{w}\right)=2(x z-x y)^{2}+1\left(z^{2}-x^{2}\right)^{2}+$ $\left(x^{2}-y^{2}\right)^{2}$. Using that

$T^{-1}(\underline{x})=\left(\left(\frac{2 d b_{i}}{a_{i}}\right)^{1 / 2 d} x_{i}\right)_{i=1}^{3}=\left(\left(\frac{2 d b_{i}}{a_{i}}\right)^{1 / 2 d} x_{i}\right)_{i=1}^{3}=\left(\left(2 b_{1}\right)^{1 / 4} x,\left(4 b_{2}\right)^{1 / 4} y,\left(4 b_{3}\right)^{1 / 4} z\right)$, where $b_{1}, b_{2}, b_{3}$ are the coefficients of $x^{4}, y^{4}, z^{4}$ in $E_{12}$, we get for

$$
E_{12}(\underline{x})=E(x)=f\left(\mathcal{H}_{3,4}, \underline{w}\right)\left(T^{-1}(\underline{x})\right),
$$

that

$$
E_{12}(\underline{x})=\frac{1}{4}\left(0.906 x^{2}-1.088 y^{2}\right)^{2}+\frac{1}{2}(-0.993 x y+0.906 x z)^{2}+\frac{1}{4}\left(-0.906 x^{2}+0.906 z^{2}\right)^{2} .
$$

With the obvious modifications, one similarly has for $E_{11}, E_{13}$ and $E_{21}$ the representations

$$
\begin{aligned}
& E_{11}=\frac{1}{2}\left(-0.799 x^{2}+0.875 x y\right)^{2}+\frac{1}{4}\left(-0.799 x^{2}+0.959 y^{2}\right)^{2} \\
& E_{13}=\frac{1}{2}\left(-0.799 z^{2}+0.875 y z\right)^{2}+\frac{1}{4}\left(-0.799 z^{2}+0.959 y^{2}\right)^{2} \\
& E_{21}=\frac{1}{2}\left(1.229 v^{2}-0.813 w^{2}\right)^{2} .
\end{aligned}
$$

This finishes step 5. The sum of all sobs representations found for the Es plus the monomial square representations for the $D$ s is a representation of the original polynomial $F$ as a sum of monomial and binomial squares as desired.

The following examples illustrate the necessity of some requirements like our hypothesis that $F$ be psd in theorem 2.9. Robinson [Ro, p272] shows that the form by later authors denoted $R$, and given by

$$
R(x, y, z)=x^{6}+y^{6}+z^{6}-\left(x^{4} y^{2}+x^{4} z^{2}+y^{4} x^{2}+y^{4} z^{2}+z^{4} x^{2}+z^{4} y^{2}\right)+3 x^{2} y^{2} z^{2}
$$

is psd but not sos. In [CL] one finds the result that each one of the ininitely many fully symmetric forms

$$
H_{\mu}(x, y, z)=\sum^{3} x^{2 \mu+4}-\sum^{6} x^{2 \mu+2} y^{2}+\sum^{3} x^{2 \mu+4} y^{2} z^{2}, \mu=1,2,3, \ldots,
$$

is psd but not sos. Since $R=H_{1}$ this is a generalization of Robinson's result. So forms can have a significant diagonal part and be psd but need not be sos. Consistent with our findings, if we change some of the signs of positive 
nondiagonal terms into their negative counterparts the obtained forms are not anymore psd.

Our main theorem, that every psd dmt form is sum of binomial squares has the consequence, of course, that every such form is sum of squares. If one gives up the requirement that these squares be binomial squares, then the number of squares can be reduced. To this end one modifies theorem 2.3 iii to ' $E$ is sos' and uses in the proof of ii $\Rightarrow$ iii the method of Reznick's paper [Re1] instead of his theory of agiforms. There he uses that the binary psd forms to which his inductive reduction of the number of variables lead, although they are sobs, can more economically be written as sums of two squares. This follows by well known reasoning using the fundamental theorem of algebra and iteratively the identity $\left(a^{2}+b^{2}\right)\left(c^{2}+d^{2}\right)=(a c-b d)^{2}+(a d+b c)^{2}$.

By the way, not every binary psd form is sobs: A representation of $f(x, y)=$ $(x-y)^{2}(x-2 y)^{2}$ as sobs would mean that each of the binomials involved must vanish when $x=y$ and also when $x=2 y$, easily seen to be impossible. For those wishing to verify the above calculations, we conclude with the list of the reals abbreviated above and increasingly ordered according to their modulus.

0.1101683087515428, 0.1122521354097604, 0.144926205793132, 0.20534577449034901, $0.2302121753633875,0.2959870826100697,0.3133395876912924,0.3288745362334108$, $0.3307276430512695,0.3433302061543539,0.4106915498069803,0.4485189743648009$, $0.4791401414414769,0.5514810256351992,0.6692723569487306,0.684485916344967$, $0.7559089941606274,0.7992831300121186,0.813299013956453,0.875784779780586$, $0.906301881060588,0.959608618892906,0.993046598277813,1.088093897804908$, $1.229560079183305,1.244091005839373$.

\section{References}

[BB] R. Bellman and E.F. Beckenbach,Inequalities, Springer 1971.

[CL] M. D. Choi, T. Y. Lam, Extremal positive semidefinite forms, Math. Ann. 231, 1-18 (1977).

[Hi] D. Hilbert, ̈̈ber die Darstellung definiter Formen als Summe von Formenquadraten, Math. Ann 32, 342-350 (1888).

[Hu] A. Hurwitz, Über den Vergleich des arithmetischen und des geometrischen Mittels, J. reine angew. Mat. 108 (1891), 266-268; in: Collected Works. II, Basel, 505-507 (1933).

[Mo] T. Motzkin, The arithmetic-geometric inequality; in Inequalities, Oved Shisha (ed.) Academic Press, 205-224 (1967).

[Po] B. Pourciau, Modern Multiplier rules, Amer. Math. Monthly, 87, 433-452 (1980). 
[Re1] B. Reznick, A quantitative version of Hurwitz' theorem on the arithmetic-geometric inequality, J. reine angew. Math. 377, 108-112 (1987).

[Re2] B. Reznick, Forms derived from the arithmetic geometric inequality, Math. Ann. 283, 431-464 (1989).

[Ro] R. Robinson, Some definite polynomials which are not sums of squares of real polynomials. In Selected questions of algebra and logic. Izdat. "Nauka" Sibirsk. Otdel. Novosibirsk, pp. 264-282 (1973).

Carla Fidalgo

Departamento de Física e Matemática, Instituto Superior de Engenharia de Coimbra, Rua Pedro Nunes, Quinta da Nora, 3030-199 Coimbra, Portugal; Department of Mathematics, University of Coimbra, 3001-454 Coimbra, Portugal

E-mail address: cfidalgo@isec.pt

Alexander KovaČEc

Department of Mathematics, University of Coimbra, 3001-454 Coimbra, Portugal

E-mail address: kovacec@mat.uc.pt 